\title{
Modeling and Analysis of Electrostatically Actuated MEMS under Combined Nonlinearities, Lorentz Force, and Mechanical Shock
}

\author{
Emran Khoshrouye Ghiasi \\ Department of Mechanical Engineering, Ferdowsi University of Mashhad, Mashhad 96911, Iran
}

\begin{abstract}
This paper shows an analysis of MEMS (micro electro mechanical systems) due to Lorentz force and mechanical shock. The formulation is based on a modified couple stress theory, the von Kármán geometric nonlinearity and Reynolds equation as well. The model contains a silicon microbeam, which is encircled by a stationary plate. The non-dimensional governing equations and associated boundary conditions are then solved iteratively through the Galerkin weighted method. The results show that pull-in voltage is dependent on the geometry nonlinearity. It is also demonstrated that by increasing voltage between the silicon microbeam and stationary plate, the pull-in instability happens.
\end{abstract}

Key words: MEMS, mechanical shock, nonlinearity, pull-in, Galerkin weighted method, dimensionless parameters.

\section{Introduction}

Development of microelectronics and IC (integrated circuit) technology are essential ingredients of MEMS (micro-electro-mechanical systems) which display low cost, fast response, mass production, and light weight [1-3]. Nowadays, researchers thoroughly have examined the behavior of MEMS, say, in relation to stability analysis of structural components compared to their similar implements. In this regard, Bagherinia et al. [4] presented a multi-physics model as well as optimization of a microbeam under electro-thermomechanical loading utilizing the second-order elastic beam theory in which the effect of the Lorentz force had been taken into account. Recently, Gkotsis et al. [5] developed an analytical model for MEMS magnetometers with consideration of the Lorentz force and piezo resistive principles. This, coupled with the fact that electrostatically actuated MEMS is basically nonlinear [6], exhibits

Corresponding author: Emran Khoshrouye Ghiasi, M.Sc. in mechanical engineering, Eng., research fields: micro/nano mechanics, mechanical vibrations, smart materials. when the nonlinear electrostatic force is greater than the elastic resonating force, the pull-in instability happens [7]. The corresponding critical voltage and displacement are known principally as the pull-in voltage and displacement, respectively. To this end, Ansari et al. [8] constructed a nonlinear model of an electrically actuated rectangular nanoplate, based on the Gurtin-Murdoch theory, for determining the residual surface stress and boundary conditions on the pull-in voltage using GDQ (generalized differential quadrature) method. Langfelder et al. [9] analyzed theoretically and experimentally the instability of capacitive MEMS accelerometers when they are subjected to a constant voltage of $\pm 1.8 \mathrm{~V}$. Xiao et al. [10] conducted a transient analys is of electrostatic actuated MEMS with piezoelectric layers based on MCST (modified couple stress theory). They have shown as a result of this trend that the voltage reduction leads to energy saving and increased reliability of the system.

Given that shock leads microstructure to hit the stationary plate, particularly during fabrication, it would be described as an applied force over a short 
period of time $[11,12]$. On the other hand, it is undeniable that bending stresses result from shock acceleration in silic on or polysilicon fabricated MEMS [13].

Typically, damping in MEMS that result from viscous dissipation of surrounding fluid has been comprised two parallel plates as movable and fixed plates [14-16]. The airflow force, in the main, is deemed to be of two major factors [17]: electrostatic force and force in free space. On the other hand, friction between viscous gas layers will have serious implications for the damping. Chu [18] demonstrated the measurement of quality factor in free space and squeeze film damping for a micro- and nano-mechanical beam resonator. On the contrary, another case was experimentally studied by De Pasquale [19]; it is found that the global error on the quality factor is computed in the range 0.19 to 0.59 percent for a rectangular silicon specimen.

This paper is divided as follows: in section 2, problem formulation to achieve nonlinear response of silicon microbeam under the Lorentz force and mechanical shock is discussed. Subsequently, the solution procedure based on the Galerkin weighted method is explained in section 3 and the results are presented in terms of numerical examples in section 4. Finally, we conclude paper in section 6.

\section{Problem State ment}

Consider a fixed-fixed microbeam with length $L$, width $b$, thickness $h$, and gap width $d$, as shown in
Fig. 1. Also, $x, y$, and $z$ are the coordinates along the length, width, and thickness, respectively, and $w(x, t)$ refers to transverse displacement of microbeam. It is true to mention that but for electrostatic actuation, microbeam would never have vibrated. In other word, by applying a voltage to the system, the microbeam will be deformed. Additionally, system is subjected to the Lorentz force and mechanical shock, and the air film between microbeam and stationary plate tends to squeeze into the environment.

\section{Governing Equations}

According to the Euler-Bernoulli beam theory [20], the displacement field can be expressed as Eq. (1)

$$
u_{1}=u-z \frac{\partial w}{\partial x}, u_{2}=0, u_{3}=w(x),
$$

where, $u$ and $w$ are the axial and transverse displacement of a point on the midplane of the microbeam, respectively. Provided microbeam encounters with small slopes after deformation, the strain tensor can be approximated by the von Kármán-type nonlinear strain as

$$
\varepsilon_{11}=\frac{\partial u_{1}}{\partial x}+\frac{1}{2}\left(\frac{\partial w}{\partial x}\right)^{2}=\frac{\partial u}{\partial x}-z \frac{\partial^{2} w}{\partial x^{2}}+\frac{1}{2}\left(\frac{\partial w}{\partial x}\right)^{2}
$$

and written in terms of Hooke's law as

$$
\sigma_{11}=E \varepsilon_{11}=E\left(\frac{\partial u}{\partial x}-z \frac{\partial^{2} w}{\partial x^{2}}+\frac{1}{2}\left(\frac{\partial w}{\partial x}\right)^{2}\right)
$$

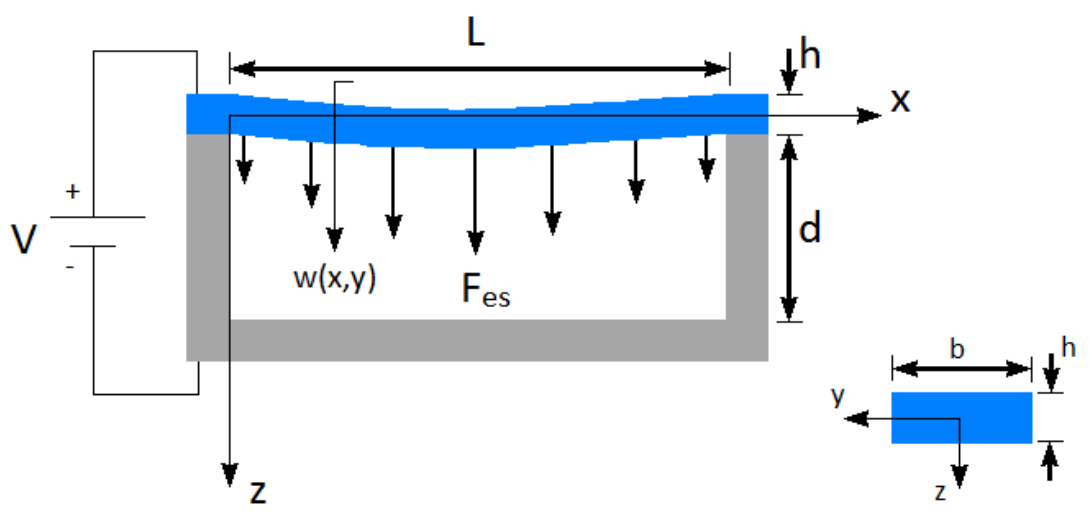

Fig. 1 A fixed-fixed microbeam with the stationary plate. 
In following based on modified couple stress theory [21], the strain energy for infinitesimal deformations is given by

$$
\bar{u}=\frac{1}{2}\left(\sigma_{i j} \varepsilon_{i j}+m_{i j} \chi_{i j}\right) \quad(i, j=1,2,3),
$$

where, $\sigma_{\mathrm{ij}}$ and $\varepsilon_{\mathrm{ij}}$ are components of the Cauchy stress and strain tensors, respectively. These tensors can be written as

$$
\begin{gathered}
m_{i j}=2 l^{2} \mu \chi_{i j}, \\
\chi_{i j}=\frac{1}{2}\left((\nabla \theta)_{i}+(\nabla \theta)_{i}^{T}\right),
\end{gathered}
$$

where, $m_{\mathrm{ij}}$ and $\chi_{\mathrm{ij}}$ are deviatoric part of couple stress and the symmetric curvature tensors, respectively, and the rotation vector is defined as

$$
\theta_{i}=\frac{1}{2}(\operatorname{curl}(\mathbf{u}))_{i} \text {. }
$$

Herein substitution of Eq. (1) into Eqs. (5)-(7) yields [22]

$$
\begin{gathered}
\theta_{2}=-\frac{\partial w}{\partial x}, \chi_{12}=\chi_{21}=-\frac{1}{2} \frac{\partial^{2} w}{\partial x^{2}}, \\
m_{12}=m_{21}=-\mu l^{2} \frac{\partial^{2} w}{\partial x^{2}} .
\end{gathered}
$$

The potential energy of microbeam and variation of the transverse loading on system can be expressed as

$$
\begin{aligned}
& U=\frac{1}{2} \int_{0}^{L} \int_{A}\left(\varepsilon_{11}\left(\sigma_{11}+\frac{N}{A}\right)+\chi_{12} m_{12}+\chi_{21} m_{21}\right) d A d x \\
& =\frac{1}{2} \int_{0}^{L} \int_{A}\left(E\left(\frac{\partial u}{\partial x}-z \frac{\partial^{2} w}{\partial x^{2}}+\left(\frac{\partial w}{\partial x}\right)^{2}\right)^{2}+\frac{N}{A}\left(\frac{\partial u}{\partial x}-z \frac{\partial^{2} w}{\partial x^{2}}+\frac{1}{2}\left(\frac{\partial w}{\partial x}\right)^{2}\right)+\mu l^{2}\left(\frac{\partial^{2} w}{\partial x^{2}}\right)^{2}\right) d A d x \\
& \delta W_{1}=\int_{0}^{L}\left(F_{e s}+F_{s h}+F_{l o}\right) \delta w d x, \\
& \delta W_{2}=\int_{0}^{L} F_{r} \delta u d x, \\
& \delta W_{3}=-\int_{0}^{L} P b \delta w d x, \\
& \rho A \frac{\partial^{2} w}{\partial t^{2}}-\frac{\partial}{\partial x}\left(E A\left(\frac{\partial u}{\partial x}+\frac{1}{2}\left(\frac{\partial w}{\partial x}\right)^{2}\right) \frac{\partial w}{\partial x}\right) \\
& +\frac{\partial^{2}}{\partial x^{2}}\left(\left(E I+\mu A I^{2}\right)\left(\frac{\partial^{2} w}{\partial x^{2}}\right)\right)= \\
& \frac{\varepsilon b V^{2}}{2(d-w)^{2}}+F_{0} g(t)+F_{l o}
\end{aligned}
$$
and pressure effect is

where, $P$ is the pressure. In accord with the principle of minimum total potential energy, one can be obtained as

$$
\delta U-\delta W_{1}-\delta W_{2}-\delta W_{3}=0 .
$$

By substitution of Eqs. (9)-(12) into Eq. (13), the nonlinear equations of motion are expressed as

$$
\rho A \frac{\partial^{2} u}{\partial t^{2}}-\frac{\partial}{\partial x}\left(E A\left(\frac{\partial u}{\partial x}+\frac{1}{2}\left(\frac{\partial w}{\partial x}\right)^{2}\right)\right)=F_{r},
$$

where, $I, \varepsilon, V$, and $g(t)$ are the moment of inertia, vacuum permittivity $\left(=8.854187 \times 10^{-12} \mathrm{~F} / \mathrm{m}\right)$, applied voltage, and the half-sine shock profile, which is defined by Brown et al. [23] as

$$
g(t)=\sin \left(\frac{\pi t}{T}\right) \underline{U}(t)+\sin \left(\frac{\pi}{T}(t-T)\right) \underline{U}(t-T),
$$

where, $T$ and $\underline{U}(t)$ are the shock duration and unit step function. 
As regards the pressure gradient through air gap is zero [24], the incompressible Reynolds equation can be written by both viscous and inertial effect within the fluid as Ref. [25].

$$
\nabla \times\left(\frac{h^{3}}{12 \mu} \rho^{\frac{1}{\eta}} \nabla P\right)=\frac{\partial}{\partial t}\left(h \rho^{\frac{1}{\eta}}\right),
$$

where, $\mu$ and $\rho$ are coefficients of viscosity and density, respectively.

The corresponding boundary conditions for fixed-fixed microbeam are expressed as

$$
\begin{gathered}
u(0, t)=0, E A\left(\frac{\partial u}{\partial x}+\frac{1}{2}\left(\frac{\partial w}{\partial x}\right)^{2}\right)_{x=L, t}=0 \\
w(0, t)=0, w(L, t)=0 \\
\left.\frac{\partial w}{\partial x}\right|_{x=0, t}=0,\left.\frac{\partial w}{\partial x}\right|_{x=L, t}=0 \\
\left.\frac{\partial P}{\partial x}\right|_{x=0, t}=0,\left.\quad \frac{\partial P}{\partial x}\right|_{x=L, t}=0
\end{gathered}
$$

Herein, it is assumed that $\rho A \frac{\partial^{2} u}{\partial t^{2}}=0$ in Eq. (14) [26]. According to the boundary conditions given in Eq. (18), one can obtain as

$$
u=-\frac{1}{2} \int_{0}^{x}\left(\frac{\partial w}{\partial x}\right)^{2} d x+\left(\frac{F_{r}}{E A}+\frac{1}{2 L} \int_{0}^{L}\left(\frac{\partial w}{\partial x}\right) d x\right) x
$$

Substituting above equation into Eq. (15) gives the equation of motion of microbeam as

$$
\begin{aligned}
& \rho A \frac{\partial^{2} w}{\partial t^{2}}+\left(E I+\mu A l^{2}\right) \frac{\partial^{4} w}{\partial x^{4}}= \\
& \frac{\partial^{2} w}{\partial x^{2}}\left(F_{r}+\frac{E A}{2 L} \int_{0}^{L}\left(\frac{\partial w}{\partial x}\right)^{2} d x\right) \\
& +\frac{\varepsilon b V^{2}}{2(d-w)^{2}}+F_{0} g(t)+F_{l o} .
\end{aligned}
$$

Considering the dimensionless parameters (listed in Table 1), dimensionless governing equations and corresponding boundary conditions of the system can be derived as

$$
\begin{gathered}
\frac{\partial^{2} W}{\partial \hat{T}^{2}}+\frac{\partial^{4} W}{\partial X^{4}}=\frac{\partial^{2} W}{\partial X^{2}}\left(N+\alpha_{1} \int_{0}^{L}\left(\frac{\partial W}{\partial X}\right)^{2} d X\right) \\
+\alpha_{2} \hat{P}+\alpha_{3} \frac{V^{2}}{(1-W)^{2}}+\alpha_{4} \hat{g}(t)+F_{l o}, \\
\frac{\partial^{2} P}{\partial X^{2}}=\alpha_{5}\left(\frac{\partial P}{\partial \hat{T}}+\frac{\partial H}{\partial \hat{T}}\right), \\
\left.\frac{\partial W}{\partial X}\right|_{x=0, t}=0,\left.\frac{\partial W}{\partial X}\right|_{x=L, t}=0 \\
\left.\frac{\partial P}{\partial X}\right|_{x=0, t}=0,\left.\frac{\partial P}{\partial X}\right|_{x=L, t}=0
\end{gathered}
$$

where, $\hat{g}(t)$ is non-dimensional half-sine shock profile.

Table 1 Dimensionless parameters.

$$
\begin{aligned}
& W=\frac{w}{d}, X=\frac{X}{L}, \hat{T}=\frac{T}{\tau}, \hat{P}=\frac{P}{p_{a}}, \tau=\sqrt{\frac{\rho b h L^{4}}{E I}}, \quad H=\frac{d}{d+w}, \alpha_{1}=6\left(\frac{h}{d_{0}}\right)^{2}, \\
& \alpha_{2}=\frac{p_{a} b L^{4}}{E I d_{0}}, \quad \alpha_{3}=\frac{6 \varepsilon L^{4}}{E d_{0}^{2} h^{3}}, \quad \alpha_{4}=\frac{12 \rho \lambda L^{4}}{E d h^{2}}, \quad \alpha_{5}=\frac{12 \mu}{p_{a} d_{0}^{2}} \sqrt{\frac{\rho b h}{E I}}, \quad N=N_{0} \frac{L^{2}}{E l} .
\end{aligned}
$$




\section{Solution Procedure}

Based on the Galerkin weighted method [27], the dimensionless deflection involved in Eq. (24) can be expressed as

$$
w(X, \hat{T})=\sum_{i=1}^{M} \varphi_{i}(X) u_{i}(\hat{T}),
$$

where, $u_{\mathrm{i}}(T)$ and $\varphi_{\mathrm{i}}(t)$ are the $i$ th generalized coordinate and the $i$ th non-dimensional linear undamped mode shape of the fixed-fixed microbeam, respectively. For simplicity, the dimensionless deflection can be written as

$$
w(X, \hat{T})=\psi(x) \zeta(\hat{T}),
$$

where, $\zeta(T)$ is the midpoint deflection and $\psi(X)$ can be determined as Ref. [28].

$$
\begin{gathered}
\psi(X)=c_{1}\left\{\cosh \left(c_{2} x\right)-\cos \left(c_{2} x\right)\right. \\
\left.-\sinh \left(c_{2} x\right)+\sin \left(c_{1} x\right)\right\},
\end{gathered}
$$

where

$$
c_{1}=0.5297, \quad c_{2}=4.4340 \text {. }
$$

It is noted that the motion of the microbeam can be simulated by solving Eqs. (24)-(31) with assuming that its zero initial condition.

\section{Results and Discussion}

In implementing the aforementioned techniques, some numerical investigations are now performed and discussed. The numerical integration of Eq. (24) is carried out until the error norm becomes less than $10^{-5}$.

In this work, it is assumed that the microbeam is made of silic on. The young modulus is presumed as $E$ $=166 \mathrm{GPa}$, which yields $\rho=2,332 \mathrm{~kg} / \mathrm{m}^{3}$ assuming Poisson's ratio as $v=0.3$ for silicon. Also, the geometric properties of the system are $b=100 \mu \mathrm{m}, h$ $=2 \mu \mathrm{m}$ and $d=2 \mu \mathrm{m}$. It should be noted that for dynamic behavior, the applied load can be calculated by substituting the Newmark's integration scheme [29] into Eq. (24) for different applied voltages.

\subsection{Example 1}

In the first example, a fixed-fixed silicon microbeam under DC applied voltage and Lorentz force is considered. In this regard, by neglecting mechanical shock involved in Eq. (24), one can obtain as

$$
\begin{aligned}
& \frac{\partial^{2} W}{\partial \hat{T}^{2}}+\frac{\partial^{4} W}{\partial X^{4}}=\frac{\partial^{2} W}{\partial X^{2}}\left(N+\alpha_{1} \int_{0}^{L}\left(\frac{\partial W}{\partial X}\right)^{2} d X\right) \\
& +\alpha_{2} \hat{P}+\alpha_{3} \frac{V^{2}}{(1-W)^{2}}+F_{l o}
\end{aligned}
$$

This example deals with the dynamic analysis of microbeam for which an analytical solution is available. The variation of applied voltage versus pull-in voltage for $L=450 \mu \mathrm{m}$ is shown in Fig. 2. It is seen that by increasing the applied voltage to the system, the microbeam softens and the corresponding pull-in voltage decreases. In order to derive a universal expression for determining the pull-in voltage without mechanical shock effects, we consider a relation between $\alpha_{2}$ and $\alpha_{3}$. Utilizing Eq. (32), we can directly determine the application range of this model by elevating the value of $\alpha_{1}$, which is dependent on the geometry parameters of the system. In general terms, to show the flexibility of the present iterative method for obtaining dynamic behavior of microbeam, the Newton-Raphson method [30] is consumed.

The result of Fig. $3 \mathrm{c}$ an be summarized to conclude that, according to Eq. (32), by introducing $\Theta$ as third term, curves increase with increase in the time. It is noted that the possibility of stability with mechanical shock in spite of Lorentz force as a provocative force is considerable. Since $-0.002 \leqslant T \leqslant+0.004$ is the domain of time in this case, at least the question could be solved by originating another domain with the aim of obtaining effect of time variable on the system. 


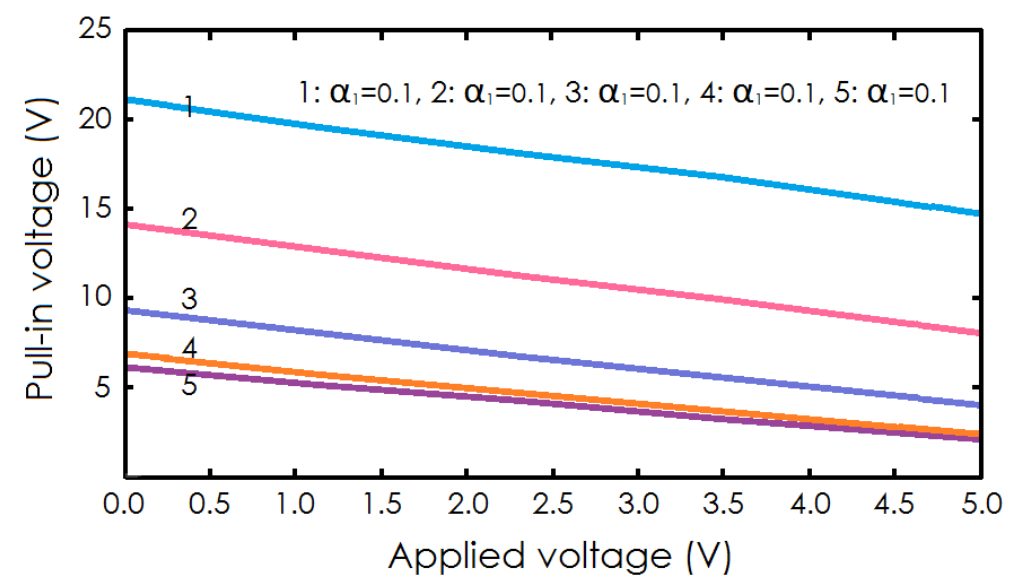

Fig. 2 Pull-in voltage values in different $\alpha_{1}$ ratios.

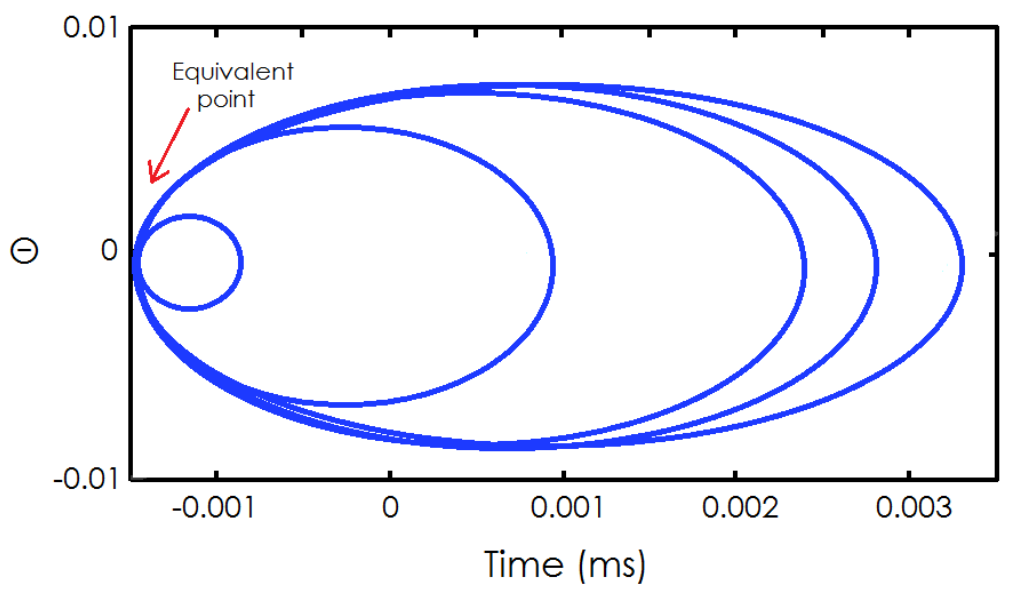

Fig. 3 Influence of time on $\Theta$ in the presence of mechanical shock.

Plus, energy dissipation is also underestimated using the Reynolds equation (Eq. (17)), which is calculated with the beam vibrating in free space.

\subsection{Example 2}

In order to validate the present method, this procedure is continued until convergence is achieved or pull-in happened. The convergence criteria is defined as

$$
\text { error }_{1}<\text { error }_{d}
$$

where,

$$
\text { error }_{1}=\sqrt{\frac{\sum_{i=1}^{n}\left|x_{i}^{m+1}-x_{i}^{m}\right|^{2}}{\sum_{i=1}^{n}\left|x_{i}^{m+1}\right|^{2}}}
$$

in which error $_{d}$ is $10^{-8}$. In this regard, Fig. 4 shows dimensionless deflection of the microbeam versus dimensionless length for $F_{r}=0$. As it is shown in this figure, the relationship of $w / d$ and $x / L$ is nonlinear and the minimum value of $w / d$ exists near the pull-in region. In particular, for the actuation voltage $V_{\mathrm{ac}}=9 \mathrm{~V}$, the system displays a nonlinear deviation abruptly. It is due to the fact that neglecting the Lorentz force may lead to a considerably underestimated $w / d$.

\subsection{Example 3}

To show the discrepancy between pull-in voltage in various actuation voltages, last example is considered as a oscillatory system with linear assumptions. It is observed that as initial gap increases, showing nearly linear increment of pull-in voltage. In this case, when 


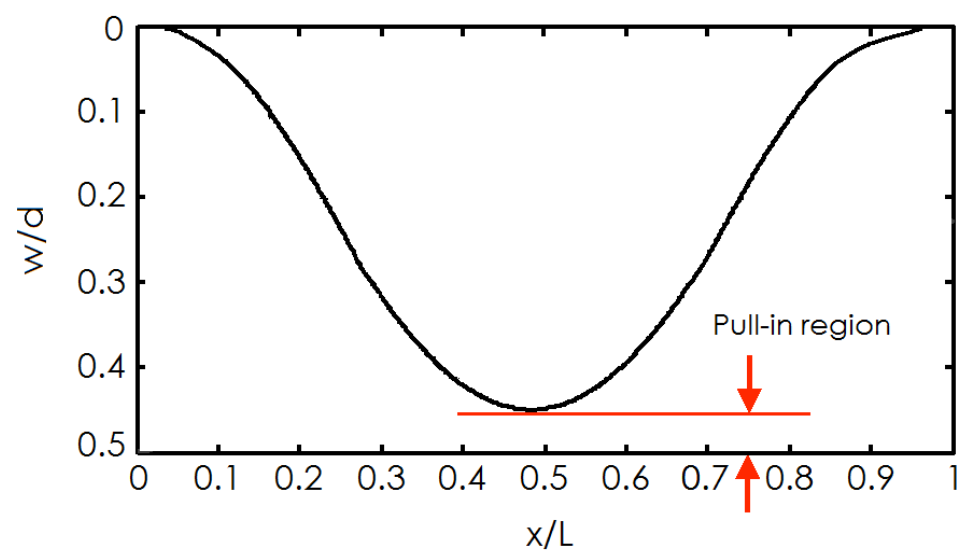

Fig. 4 Influence of $x / L$ change on $w / d$ where $V_{\mathrm{ac}}=9 \mathrm{~V}$.

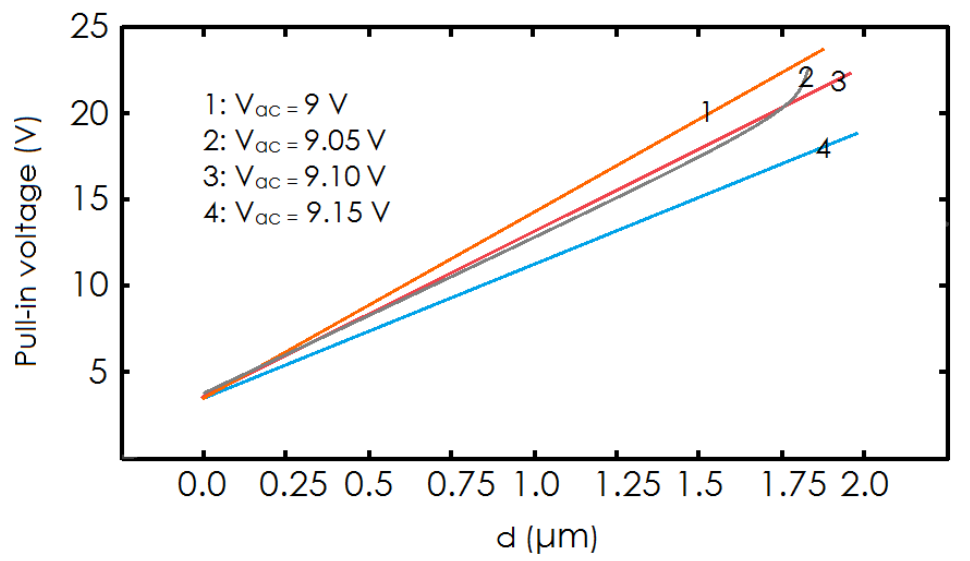

Fig. 5 Convergence of pull-in voltage versus $d$ in various $V_{\text {ac }}$.

actuation voltage is $V_{\text {ac }}=9.05 \mathrm{~V}$ (or near $9.05 \mathrm{~V}$ ), the behavior of system differs from others (see Fig. 5). It is due to the fact that the effect of electrostatic actuation to reduce the structural stability is undeniable.

\section{Conclusion}

In this paper, MCST in conjunction with the effect of Lorentz force and mechanical shock has been introduced to investigate nonlinear dynamic response of electrostatically actuated MEMS. To this aim, Reynolds equation was also used to obtain the effects of pressure between two plates. Afterwards the model was solved using the Galerkin weighted method. Finally it is indicated that as the ratio of dimensionless deflection to initial gap decreases, the results converge to the pull-in instability. Furthermore, the pull-in voltage decreases with applied voltage increasing.

\section{References}

[1] Dewey, A., Srinivasan, V., and Icoz, E. 2001. "Visual Modeling and Design of Micro-electro-mechanical Systems Transducers." Microelectronics Journal 32: 373-81.

[2] Sheng, H., and Zhang, T. 2015. "MEM S-Based Low-Cost Strap-Down AHRS Research." Measurement 59: 63-72.

[3] Spearing, S. M. 2000. "Materials Issues in Microelectromechanical Systems." Acta Material 48: 179-96.

[4] Bagherinia, M., Bruggi, M., Corigliano, A., Mariani, S., and Lasalandra, E. 2014. "Geometry Optimization of a Lorentz Force, Resonating MEMS Magnetometer." Microelectronics Reliability 54: 1192-9.

[5] Gkotsis, P., Castro, M. L., Huerta, F. L., May, A. L. H., and Raskin, J. P. 2015. "Mechanical Characterization and Modeling of Lorentz Force Based MEMS Magnetic Field Sensors." Solid-State Electronics 112: 68-77.

[6] Chuang, W. C., Lee, H. L., Chang, P. Z., and Hu, Y. C. 

Force, and Mechanical Shock

2010. "Review on the Modeling of Electrostatic MEMS." Sensors 10: 6149-71.

[7] Sadeghian, H., Rezazadeh, G., and Osterberg, P. M. 2007. "Application of the Generalized Differential Quadrature Method to the Study of Pull-in Phenomena of MEMS Switches." Journal of Microelectromechanical Systems 16 (6): 1334-40.

[8] Asghari, R., Mohammadi, V., Shojaei, M. F., Gholami, R., and Darabi, M. A. 2014. "A Geometrically Non-Linear Plate Model Including Surface Stress Effect for the Pull-in Instability Analysis of Rectangular Nanoplates under Hydrostatic and Electrostatic Actuations." International Journal of Non-Linear Mechanics 67: 16-26.

[9] Langfelder, G., Frizzi, T., Longoni, A., Tocchio, A., Manelli, D., and Lasalandra, E. 2011. "Readout of MEMS Capacitive Sensors beyond the Condition of Pull-in Instability." Sensors and Actuators A 167: 374-84.

[10] Xiao, Y., Wang, B., and Zhou, S. 2015. "Pull-in Voltage Analysis of Electrostatically Actuated MEMS with Piezoelectric Layers: A Size-Dependent Model." Mechanics Research Communications 66: 7-14.

[11] Fathalilou, M., Motallebi, A., Yagubizadeh, H., Rezazadeh, G., Shirazi, K., and Alizadeh, Y. 2009. "Mechanical Behavior of an Electrostatically-Actuated Microbeam under Mechanical Shock." Journal of Solid Mechanics 1: 45-57.

[12] Younis, M. I., Alsaleem, F., and Jordy, D. 2007. "The Response of Clamped-Clamped Microbeams under Mechanical Shock." International Journal of Non-Linear Mechanics 42: 643-57.

[13] Millet, O., Collard, D., and Buchaillot, L. 2002. "Reliability of Packaged MEMS in Shock Environment: Crack and Stiction Modeling." In Proceedings of SPIE-The International Society for Optical Eng ineering, 696-703.

[14] Pasquale, G. D., and Somà, A. 2015. "An Energetic Approach for the Experimental Identification of Damping in Microstructures." Mechanical Systems and Signal Processing 50-51: 338-48.

[15] Guo, X., and Yi, Y. B. 2014. "Suppression of Thermoelastic Damping in MEMS Beam Resonator by Piezoresistivity." Journal of Sound and Vibration 333: 1079-95.

[16] Homentcovschi, D., Murray, B. T., and Miles, R. N. 2013. "Viscous Damping and Spring Force Calculation of
Regularly Perforated MEMS Microstructures in the Stokes' Approximation." Sensors and Actuators A 201: 281-8.

[17] Hosaka, H., Itao, K., and Kuroda, S. 1995. "Damping Characteristics of Beam-Shaped Micro-Oscillators." Sensors and Actuators A 49: 87-95.

[18] Chu, H. M. 2016. "Air Damping Models for Micro- and Nano-Mechanical Beam Resonators in Molecular-Flow Regime." Vacuum 126: 45-50.

[19] Pasquale, G. D. 2013. "Experimental Analy sis of Viscous and Material Damping in Microstructures through the Interferometric Microscopy Technique with Climatic Chamber." Journal of Sound and Vibration 332: 4103-21.

[20] Reddy, J. N. 2007. Theory and Analysis of Elastic Plates and Shells. Second Edition, Taylor \& Francis.

[21] Yang, F., Chong, A. C. M., Lam, D. C. C., and Tong, P. 2002. "Couple Stress Based Strain Gradient Theory for Elasticity." International Journal of Solids and Structures 39: 2731-43.

[22] Park, S. K., and Gao, X. L. 2006. "Bernoulli-Euler Beam Model Based on a Modified Couple Stress Theory." Journal of Micromechanics and Microengineering 16: 2355-9.

[23] Brown, T. G., Davis, B., Hepner, D., Faust, J., Myers, C., Muller, P., Harkins, T., Hollis, M., Miller, C., and Placzankis, B. 2001. "Strap-Down Microelectromechanical Sensors for High-G Munition Applications.” Transactions on Magnetics 37 (1): 336-42.

[24] Darling, R. B., Hivick, C., and Xu, J. 1998. "Compact Analytical Modeling of Squeeze Film Damping with Arbitrary Venting Conditions Using a Green's Function Approach." Sensors and Actuators A 70: 32-41.

[25] Blech, J. J. 1983. "On Isothermal Squeeze Films." Journal of Lubrication Technology 105 : 615-20.

[26] Younis, M. I. 2011. MEMS Linear and Nonlinear Statics and Dynamics. Springer.

[27] Reddy, J. N. 2002. Energy Principles and Variational Methods in Applied Mechanics. John Wiley \& Sons.

[28] Balachandran, E., and Magrab, E. 2009. Vibrations Second Edition, Cengage Learning.

[29] Rubin, M. B. 2007. “A Simplified Implicit Newmark Integration Scheme for Finite Rotations." Computers and Mathematics with Applications 53: 219-31.

[30] Sheng, D., Sloan, S. W., and Abbo, A. J. 2002. "An Automatic Newton-Raphson Scheme." International Journal of Geomechanics 2 (4): 471-502. 